\title{
Reducing Response-Time Bounds for DAG-Based Task Systems on Heterogeneous Multicore Platforms
}

\author{
Kecheng Yang, Ming Yang, and James H. Anderson \\ Department of Computer Science \\ University of North Carolina at Chapel Hill \\ \{yangk,yang,anderson\}@cs.unc.edu
}

\begin{abstract}
This paper considers for the first time end-to-end response-time analysis for DAG-based real-time task systems implemented on heterogeneous multicore platforms. The specific analysis problem that is considered was motivated by an industrial collaboration involving wireless cellular base stations. The DAG-based systems considered herein allow intra-task parallelism: while each invocation of a task (i.e., DAG node) is sequential, successive invocations of a task may execute in parallel. In the proposed analysis, this characteristic is exploited to reduce response-time bounds. Additionally, there is some leeway in choosing how to set tasks' relative deadlines. It is shown that by resolving such choices holistically via linear programming, response-time bounds can be further reduced. Finally, in the considered use case, DAGs are defined based upon just a few templates and individually often have quite low utilizations. It is shown that, by combining many such DAGs into one of higher utilization, response-time bounds can often be drastically lowered. The effectiveness of these techniques is demonstrated via both casestudy and schedulability experiments.
\end{abstract}

\section{INTRODUCTION}

The multicore revolution is currently undergoing a second wave of innovation in the form of heterogeneous hardware platforms. In the domain of real-time embedded systems, such platforms may be desirable to use for a variety of reasons. For example, ARM's big.LITTLE multicore architecture [8] enables performance and energy concerns to be balanced by providing a mix of relatively slower, low-power cores and faster, high-power ones. Unfortunately, the move towards greater heterogeneity is further complicating software design processes that were already being challenged on account of the significant parallelism that exists in "conventional" multicore platforms with identical processors. Such complications are impeding advancements in the embedded computing industry today.

\footnotetext{
*Work supported by NSF grants CNS 1563845, CPS 1239135 , CNS 1409175, and CPS 1446631, AFOSR grant FA9550-14-10161, ARO grant W911NF-14-1-0499, and funding from both General Motors and FutureWei Corp.

Permission to make digital or hard copies of all or part of this work for personal or classroom use is granted without fee provided that copies are not made or distributed for profit or commercial advantage and that copies bear this notice and the full citation on the first page. Copyrights for components of this work owned by others than the author(s) must be honored. Abstracting with credit is permitted. To copy otherwise, or republish, to post on servers or to redistribute to lists, requires prior specific permission and/or a fee. Request permissions from permissions@ acm.org.

RTNS '16, October 19 - 21, 2016, Brest, France
}

(C) 2016 Copyright held by the owner/author(s). Publication rights licensed to ACM. ISBN 978-1-4503-4787-7/16/10 . .\$15.00

DOI: http://dx.doi.org/10.1145/2997465.2997486
Problem considered herein. In this paper, we report on our efforts towards solving a particular real-time analysis problem concerning heterogeneity motivated by an industrial collaboration. This problem pertains to the processing done by cellular base stations in wireless networks. Due to space constraints, we refrain from delving into specifics regarding this particular application domain, opting instead for a more abstract treatment of the problem at hand.

This problem involves the scheduling of real-time dataflows on heterogeneous computational elements (CEs), such as CPUs, digital signal processors (DSPs), or one of many types of hardware accelerators. Each dataflow is represented by a DAG, the nodes (resp., edges) of which represent tasks (resp., producer/consumer relationships). A given task is restricted to run on a specific CE type. Task preemption may be impossible for some CEs and should in any case be discouraged. Each DAG has a single source task that is invoked periodically. Intra-task parallelism is allowed in the sense that consecutive jobs (i.e., invocations) of the same task can execute in parallel (but each job executes sequentially). In fact, a later job can finish earlier due to variations in running times. ${ }^{1}$ The DAGs to be supported are defined using a relatively small number of "templates," i.e., many DAGs may exist that are structurally identical. The challenge is to devise a multi-resource, real-time scheduler for supporting dataflows as described here with accompanying per-dataflow end-to-end response-time analysis. That is, an upper bound on the response time of a single invocation of each DAG in a system comprised of such DAGs is required.

Related work. The literature on real-time systems includes much work pertaining to the scheduling of DAG-based task systems on identical multiprocessor platforms $[1,2,4,5,6,7,9,10,13,15,17$, $18,19,20,21,22,23,24]$. However, we are not aware of any corresponding work directed at heterogeneous platforms. Moreover, the problem above has facets-such as allowing intra-task parallelism and defining potentially many DAGs based upon relatively few templates-that have not been fully explored. (Intra-task parallelism has been considered in a limited context in DAG-related work pertaining to identical multiprocessors [7, 9, 23].) Additionally, in prior work on the real-time scheduling of task systemswhich are not DAG-based-upon heterogeneous platforms, taskto-CE assignments have been a paramount concern. In our setting, this is a non-issue, as this assignment is pre-determined based on CE functionalities.

Beyond the real-time systems community, DAG-based systems implemented on heterogeneous platforms have been considered before $($ e.g., $[3,16,26])$. However, all such work known to us focuses on one-shot, aperiodic DAG-based jobs, rather than periodic

\footnotetext{
${ }^{1}$ In the considered application domain, the data produced by subsequent jobs can be buffered until all prior jobs of the same task have completed.
} 
or sporadic DAG-based task systems. Moreover, real-time issues are considered only obliquely from the perspectives of job admission control or job makespan minimization.

Contributions. In this paper, we formalize the problem described above and then address it by proposing a scheduling approach and associated end-to-end response-time analysis. In the first part of the paper, we attack the problem by presenting a transformation process whereby successive task models are introduced such that: (i) the first task model directly formalizes the problem above; (ii) prior analysis can be applied to the last model to obtain response-time bounds under earliest-deadline-first (EDF) scheduling; and (iii) each successive model is a refinement of the prior one in the sense that all DAG-based precedence constraints are preserved. Such a transformation approach was previously used by Liu and Anderson [20] in work on DAG-based systems, but that work focused on identical multiprocessors. Moreover, our work differs from theirs in that we allow intra-task parallelism. This enables much smaller end-to-end response-time bounds to be derived.

After presenting this transformation process, we discuss two techniques that can reduce the response-time bounds enabled by this process. The first technique exploits the fact that some leeway exists in setting tasks' relative deadlines. By setting more aggressive deadlines for tasks along "long" paths in a DAG, the overall end-to-end response-time bound of that DAG can be reduced. We show that such deadline adjustments can be made by solving a linear program.

The second technique exploits the fact that, in the considered context, DAGs are defined using relatively few templates and typically have quite low utilizations. These facts enable us to reduce response-time bounds by combining many DAGs into one of larger utilization. As a very simple example, two DAGs with a period of 10 time units might be combined into one with a period of 5 time units. A response-time-bound reduction is enabled because these bounds tend to be proportional to periods. In the considered application domain, the extent of combining can be much more extensive: upwards of 40 DAGs may be combinable.

As a final contribution, we evaluate our proposed techniques via case-study and schedulability experiments. These experiments show that our techniques can significantly reduce response-time bounds. Furthermore, our analysis supports "early releasing" [12] (see Sec. 7) to improve observed end-to-end response times. We experimentally demonstrate the efficacy of this as well.

Organization. In the rest of this paper, we formalize the considered problem (Sec. 2), present the refinements mentioned above that enable the use of prior analysis (Secs. 3-4), show that the bounds arising from this analysis can be improved via linear programming (Sec. 5) and DAG combining (Sec. 6), discuss early releasing (Sec. 7), present our case-study (Sec. 8) and schedulability (Sec. 9) experiments, and conclude (Sec. 10).

\section{SYSTEM MODEL}

In this section, we formalize the dataflow-scheduling problem described in Sec. 1 and introduce relevant terminology. Each dataflow is represented by a DAG, as discussed earlier.

We specifically consider a system $G=\left\{G_{1}, G_{2}, \ldots, G_{N}\right\}$ comprised of $N$ DAGs. The DAG $G_{i}$ consists of $n_{i}$ nodes, which correspond to $n_{i}$ tasks, denoted $\tau_{i}^{1}, \tau_{i}^{2}, \ldots, \tau_{i}^{n_{i}}$. Each task $\tau_{i}^{v}$ releases a (potentially infinite) sequence of jobs $J_{i, 1}^{v}, J_{i, 2}^{v}, \ldots$. The edges in $G_{i}$ reflect producer/consumer relationships. A particular task $\tau_{i}^{v}$ 's producers are those tasks with outgoing edges directed to $\tau_{i}^{v}$, and its consumers are those with incoming edges directed from $\tau_{i}^{v}$.
The $j^{\text {th }}$ job of task $\tau_{i}^{v}, J_{i, j}^{v}$, cannot commence execution until the $j^{\text {th }}$ jobs of all of its producers have completed; this ensures that its necessary input data is available. Such job dependencies only exist with respect to the same invocation of a DAG, and not across different invocations. That is, while jobs must execute sequentially, intra-task parallelism is allowed.

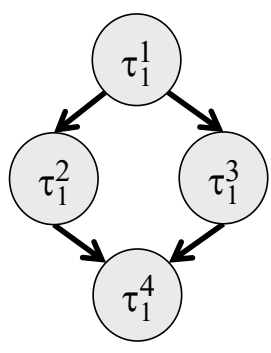

Example 1. Fig. 1 shows an example DAG, $G_{1}$. Task $\tau_{1}^{4}$ 's producers are tasks $\tau_{1}^{2}$ and $\tau_{1}^{3}$, thus for any $j, J_{1, j}^{4}$ needs input data from each of $J_{1, j}^{2}$ and $J_{1, j}^{3}$, so it must wait until those jobs complete. Because intra-task parallelism is allowed, $J_{1, j}^{4}$ and $J_{1, j+1}^{4}$ could potentially execute in parallel.

To simplify analysis, we assume that each DAG $G_{i}$ has exactly one source task $\tau_{i}^{1}$, which has only outgoing edges, and one $\operatorname{sink} \operatorname{task} \tau_{i}^{n_{i}}$, which has only incoming edges. Multi-source/multisink DAGs can be supported with the addition of singular "virtual" sources and sinks that connect multiple sources and sinks, respectively. Virtual sources and sinks have a worst-case execution time (WCET) of zero.

We consider the scheduling of DAGs as just described on a heterogeneous hardware platform consisting of different types of CEs. A given CE might be a CPU, DSP, or some specialized hardware accelerator (HAC). The CEs are organized in $M \mathrm{CE}$ pools, where each CE pool $\pi_{k}$ consists of $m_{k}$ identical CEs. Each task $\tau_{i}^{v}$ has a parameter $P_{i}^{v}$ that denotes the particular CE pool on which it must run, i.e., $P_{i}^{v}=\pi_{k}$ means that each job of $\tau_{i}^{v}$ must be scheduled on a CE in the CE pool $\pi_{k}$. The WCET of task $\tau_{i}^{v}$ is denoted $C_{i}^{v}$.

Although the problem description in Sec. 1 indicated that source tasks are released periodically, we generalize this to allow sporadic releases, i.e., for the DAG $G_{i}$, the job releases of $\tau_{i}^{1}$ have a minimum separation time, denoted $T_{i}$. A non-source task $\tau_{i}^{v}(v>1)$ releases its $j^{\text {th }}$ job $J_{i, j}^{v}$ when the $j^{\text {th }}$ jobs of all its producer tasks in $G_{i}$ have completed. That is, letting $a_{i, j}^{v}$ and $f_{i, j}^{v}$ denote the release (or arrival) and finish times of $J_{i, j}^{v}$, respectively,

$$
a_{i, j}^{v}=\max \left\{f_{i, j}^{w} \mid \tau_{i}^{w} \text { is a producer of } \tau_{i}^{v}\right\} .
$$

The response time of job $J_{i, j}^{v}$ is defined as $f_{i, j}^{v}-a_{i, j}^{v}$, and the endto-end response time of the DAG $G_{i}$ as $f_{i, j}^{n_{i}}-a_{i, j}^{1}$.

Example 2. Fig. 2 depicts an example schedule for the DAG $G_{1}$ in Fig. 1, assuming task $\tau_{1}^{2}$ is required to execute on a DSP and the other tasks are required to execute on a CPU. The first (resp., second) job of each task has a lighter (resp., darker) shading to make them easier to distinguish. Tasks $\tau_{1}^{2}$ and $\tau_{1}^{3}$ have only one producer, $\tau_{1}^{1}$, so when task $\tau_{1}^{1}$ finishes a job at times 3 and $8, \tau_{1}^{2}$ and $\tau_{1}^{3}$ release a job immediately. In contrast, task $\tau_{1}^{4}$ has two producers, $\tau_{1}^{2}$ and $\tau_{1}^{3}$. Therefore, $\tau_{1}^{4}$ cannot release a job at time 5 when $\tau_{1}^{3}$ finishes a job, but rather must wait until time 6 when $\tau_{1}^{2}$ also finishes a job. Note that consecutive jobs of the same task might execute in parallel (e.g., $J_{1,1}^{4}$ and $J_{1,2}^{4}$ execute in parallel during $[11,12)$ ). Furthermore, for a given task, a later-released job (e.g., $\left.J_{1,2}^{4}\right)$ may even finish earlier than an earlier-released one (e.g., $\left.J_{1,1}^{4}\right)$ due to execution-time variations.

Scheduling. Since many CEs are non-preemptible, we use the non-preemptive global EDF (G-EDF) scheduling algorithm within each CE pool. The deadline of job $J_{i, j}^{v}$ is given by

$$
d_{i, j}^{v}=a_{i, j}^{v}+D_{i}^{v}
$$




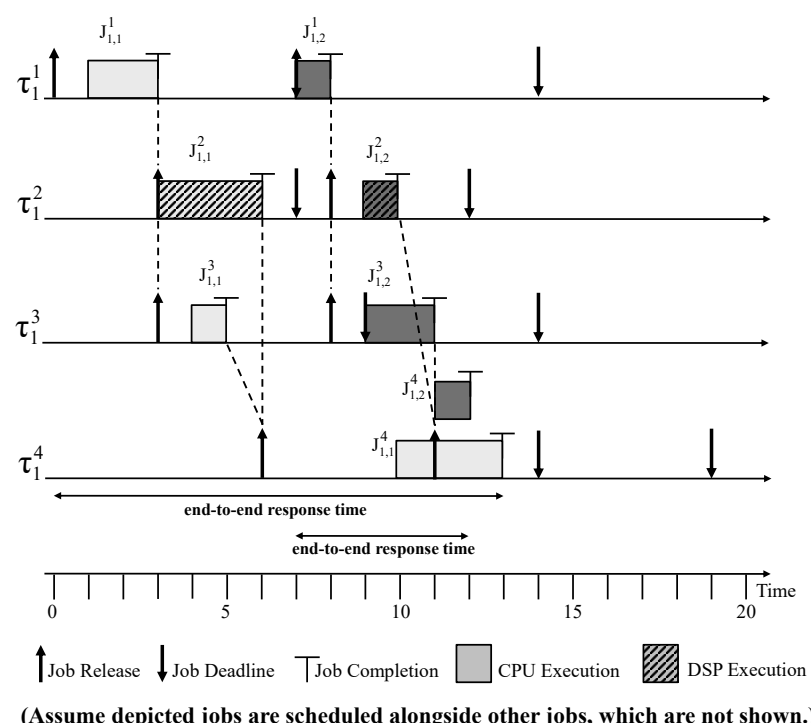

Figure 2: Example schedule for the DAG in $G_{1}$ in Fig. 1.

where $D_{i}^{v}$ is the relative deadline of task $\tau_{i}^{v}$. For example, in the example schedule in Fig. 2, relative deadlines of $D_{1}^{1}=7, D_{1}^{2}=4$, $D_{1}^{3}=6$, and $D_{1}^{4}=8$ are assumed.

In the context of this paper, deadlines mainly serve the purpose of determining jobs' priorities rather than strict timing constraints for individual jobs. Therefore, deadline misses are acceptable as long as the end-to-end response time of each DAG can be reasonably bounded.

Utilization. We denote the utilization of task $\tau_{i}^{v}$ by

$$
u_{i}^{v}=\frac{C_{i}^{v}}{T_{i}} .
$$

We also use $\Gamma_{k}$ to denote the set of tasks that are required to execute on the $\mathrm{CE}$ pool $\pi_{k}$, i.e.,

$$
\Gamma_{k}=\left\{\tau_{i}^{v} \mid P_{i}^{v}=\pi_{k}\right\}
$$

The overutilization of a CE pool could cause unbounded response times, so we require for each $k$,

$$
\sum_{\tau_{i}^{v} \in \Gamma_{k}} u_{i}^{v} \leq m_{k} .
$$

\section{OFFSET-BASED INDEPENDENT TASKS}

In this section, we present a second task model, which is a refinement of that just presented, as will be shown in Sec. 4. The prior model is somewhat problematic because of difficult-to-analyze dependencies among jobs. In particular, by (1), the release times of jobs of non-source tasks depend on the finish times of other jobs, and hence on their execution times. By (2), deadlines (and hence priorities) of jobs are affected by similar dependencies.

In order to ease analysis difficulties associated with such job dependencies, we introduce here the offset-based independent task (obi-task) model. Under this model, tasks are partitioned into groups. The $i^{\text {th }}$ such group consists of tasks denoted $\tau_{i}^{1}, \tau_{i}^{2}, \ldots, \tau_{i}^{n_{i}}$, where $\tau_{i}^{1}$ is a designated source task that releases jobs sporadically with a minimum separation of $T_{i}$. That is, for any positive integer $j$,

$$
a_{i, j+1}^{1}-a_{i, j}^{1} \geq T_{i}
$$

Job releases of each non-source task $\tau_{i}^{v}$ are governed by a new parameter $\Phi_{i}^{v}$, called the offset of $\tau_{i}^{v}$. Specifically, $\tau_{i}^{v}$ releases its $j^{\text {th }}$ job exactly $\Phi_{i}^{v}$ time units after the release time of the $j^{\text {th }}$ job of the source task $\tau_{i}^{1}$ of its group. That is,

$$
a_{i, j}^{v}=a_{i, j}^{1}+\Phi_{i}^{v} .
$$

For consistency, we define

$$
\Phi_{i}^{1}=0
$$

Under the obi-task model, a job of a task $\tau_{i}^{v}$ can be scheduled at any time after its release independently of the execution of any other jobs, even jobs of the same task $\tau_{i}^{v}$.

The definitions so far have dealt with job releases. Additionally, the two per-task parameters $C_{i}^{v}$ and $P_{i}^{v}$ from Sec. 2 are retained with the same definitions.

The following property shows that every obi-task $\tau_{i}^{v}$ has a minimum job-release separation of $T_{i}$.

Property 1. For any obi-task $\tau_{i}^{v}, a_{i, j+1}^{v}-a_{i, j}^{v} \geq T_{i}$.

Proof.

$$
\begin{aligned}
a_{i, j+1}^{v}-a_{i, j}^{v}= & \{\text { by (7) }\} \\
& \left(a_{i, j+1}^{1}+\Phi_{i}^{v}\right)-\left(a_{i, j}^{1}+\Phi_{i}^{v}\right) \\
\geq & \{\text { by (6) }\} \\
& T_{i}
\end{aligned}
$$

\section{RESPONSE-TIME BOUNDS}

In this section, we establish two results that enable prior work to be leveraged to establish response-time bounds for DAG-based task systems. First, we show that, under the obi-task model with arbitrary offset settings, per-task response-time bounds can be derived by exploiting prior work pertaining to a task model called the npc-sporadic task model ("npc" stands for "no precedence constraints" - this refers to the lack of precedence constraints among jobs of the same task) [14, 27]. Second, we show that, by properly setting offsets, any DAG-based task system can be transformed to a corresponding obi-task system.

\subsection{Response-Time Bounds for Obi-Tasks}

An $n p c$-sporadic task $\tau_{i}$ is specified by $\left(C_{i}, T_{i}, D_{i}\right)$, where $C_{i}$ is its WCET, $T_{i}$ is the minimum separation time between consecutive job releases of $\tau_{i}$, and $D_{i}$ is its relative deadline. As before, $\tau_{i}$ 's utilization is $u_{i}=C_{i} / T_{i}$.

The main difference between the conventional sporadic task model and the npc-sporadic task model is that the former requires successive jobs of each task to execute in sequence while the latter allows them to execute in parallel. That is, under the conventional sporadic task model, job $J_{i, j+1}$ cannot commence execution until its predecessor $J_{i, j}$ completes, even if $a_{i, j+1}$, the release time of $J_{i, j+1}$, has elapsed. In contrast, under the npc-sporadic task model, any job can execute as soon as it is released. Note that, although we allow intra-task parallelism, each individual job still must execute sequentially.

Yang and Anderson [27] investigated the G-EDF scheduling of npc-sporadic tasks on uniform heterogeneous multiprocessor platforms where different processors may have different speeds. By setting each processor's speed to be 1.0, the following theorem follows from their work.

Theorem 1. (Follows from Theorem 4 in [27]) Consider the scheduling of a set of npc-sporadic tasks $\tau$ on $m$ identical multiprocessors. Under non-preemptive G-EDF, each npc-task $\tau_{i} \in \tau$ 
has the following response-time bound, provided $\sum_{\tau_{l} \in \tau} u_{l} \leq m$.

$$
\begin{array}{r}
\frac{1}{m}\left(D_{i} \cdot \sum_{\tau_{l} \in \tau} u_{l}+\sum_{\tau_{l} \in \tau}\left(u_{l} \cdot \max _{\tau_{l} \in \tau}\left\{0, T_{l}-D_{l}\right\}\right)\right) \\
+\max _{\tau_{l} \in \tau}\left\{C_{l}\right\}+\frac{m-1}{m} C_{i} .
\end{array}
$$

We now show that Theorem 1 can be applied to obtain per-task response-time bounds for any obi-task set.

Concrete vs. non-concrete. A concrete sequence of job releases that satisfies a task's specification (under either the obi- or npcsporadic task model) is called an instantiation of that task. An instantiation of a task set is defined similarly. In contrast, a task or a task set that can have multiple (potentially infinite) instantiations satisfying its specification (e.g., minimum release separation) is called non-concrete.

By Property 1, any instantiation of an obi-task $\tau_{i}^{v}$ is an instantiation of the npc-sporadic task $\tau_{i}^{v}=\left(C_{i}^{v}, T_{i}, D_{i}^{v}\right)$. Hence, any instantiation of an obi-task set $\left\{\tau_{i}^{v} \mid P_{i}^{v}=\pi_{k}\right\}$ is an instantiation of the npc-sporadic task set $\left\{\tau_{i}^{v} \mid P_{i}^{v}=\pi_{k}\right\}$. Also, since obi-tasks execute independently of one another, obi-tasks executing in different CE pools cannot affect each other. Since each CE pool $\pi_{k}$ has $m_{k}$ identical processors, the problem we must consider is that of scheduling an instantiation of the npc-sporadic task set $\left\{\tau_{i}^{v} \mid P_{i}^{v}=\pi_{k}\right\}$ on $m_{k}$ identical processors. Since Theorem 1 applies to a non-concrete npc-sporadic task set, it applies to every concrete instantiation of such a task set. Thus, we have the following response-time bound for each obi-task $\tau_{i}^{v}$ :

$$
\begin{aligned}
R_{i}^{v}= & \frac{1}{m_{k}}\left(D_{i}^{v} \cdot \sum_{\tau_{l}^{w} \in \Gamma_{k}} u_{l}^{w}+\sum_{\tau_{l}^{w} \in \Gamma_{k}}\left(u_{l}^{w} \cdot \max \left\{0, T_{l}-D_{l}^{w}\right\}\right)\right) \\
& +\max _{\tau_{l}^{w} \in \Gamma_{k}}\left\{C_{l}^{w}\right\}+\frac{m_{k}-1}{m_{k}} C_{i}^{v}
\end{aligned}
$$

where $\Gamma_{k}=\left\{\tau_{l}^{w} \mid P_{l}^{w}=\pi_{k}\right\}$.

Note that (9) is applicable as long as all relative deadlines are non-negative, and applies assuming any arbitrary offset setting.

\subsection{From DAG-Based Task Sets to Obi-Task Sets}

We now show that, by properly setting offsets, any DAG-based task set can be transformed to an obi-task set, and per-DAG end-toend response-time bounds can be derived by leveraging the obi-task response-time bounds just stated.

Any DAG-based task set can be implemented by an obi-task set in an obvious way: each DAG becomes an obi-task group with the same task designated as its source, and all $T_{i}, C_{i}^{v}$, and $P_{i}^{v}$ parameters are retained without modification. What is less obvious is how to define task offsets under the obi-task model. This is done by setting each $\Phi_{i}^{v}(v \neq 1)$ parameter to be a constant such that

$$
\Phi_{i}^{v} \geq \max _{\tau_{i}^{k} \in \operatorname{prod}\left(\tau_{i}^{v}\right)}\left\{\Phi_{i}^{k}+R_{i}^{k}\right\},
$$

where $\operatorname{prod}\left(\tau_{i}^{v}\right)$ denotes the set of obi-tasks corresponding to the DAG-based tasks that are the producers of the DAG-based task $\tau_{i}^{v}$ in $G_{i}$, and $R_{i}^{k}$ denotes a response-time bound for the obi-task $\tau_{i}^{k}$. For now, we assume that $R_{i}^{k}$ is known, but later, we will show how to compute it.

Example 3. Consider again the DAG $G_{1}$ in Fig. 1. Assume that, after applying the above transformation, the obi-tasks have responsetime bounds of $R_{1}^{1}=9, R_{1}^{2}=5, R_{1}^{3}=7$, and $R_{1}^{4}=9$, respec-

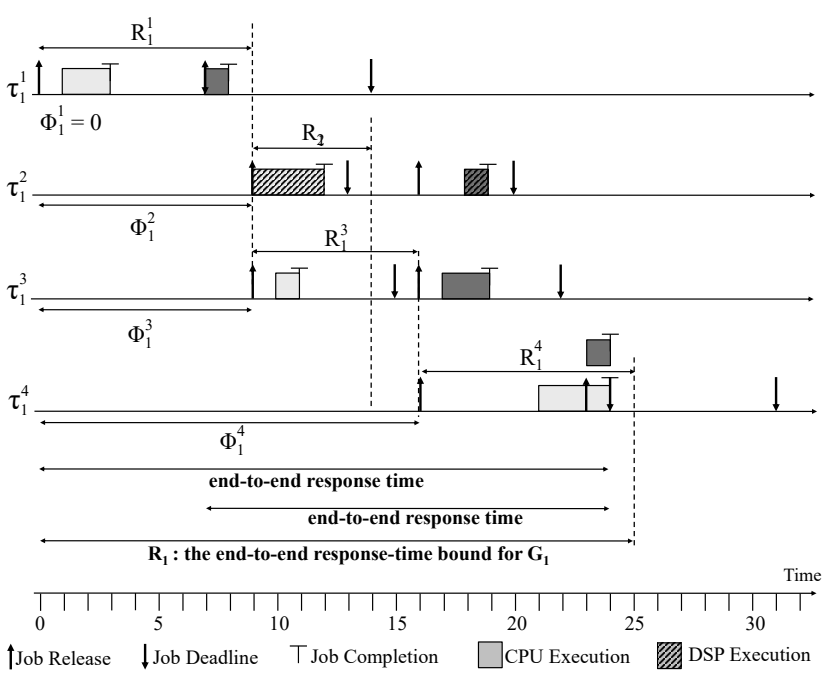

(Assume depicted jobs are scheduled alongside other jobs, which are not shown.)

Figure 3: Example schedule of the obi-tasks corresponding to the DAGbased tasks in $G_{1}$ in Fig. 1.

tively. Then, we can set $\Phi_{1}^{1}=0, \Phi_{1}^{2}=9, \Phi_{1}^{3}=9$, and $\Phi_{1}^{4}=16$, respectively, and satisfy (10). With these response-time bounds, the end-to-end response-time bound that can be guaranteed is determined by $R_{1}^{1}, R_{1}^{3}$, and $R_{1}^{4}$ and is given by $R_{1}=25$. Fig. 3 depicts a possible schedule for these obi-tasks and illustrates the transformation. Like in Fig. 2, the first (resp., second) job of each task has a lighter (resp., darker) shading, and intra-task parallelism is possible (e.g., $J_{1,1}^{4}$ and $J_{1,2}^{4}$ in time interval $[23,24)$ ).

The following properties follow from this transformation process. According to Property 2, a DAG-based task set can be implemented by a corresponding set of obi-tasks, and all producer/consumer constraints in the DAG-based specification will be implicitly guaranteed, provided the offsets of the obi-tasks are properly set (i.e., satisfy (10))

Property 2. If $\tau_{i}^{k}$ is a producer of $\tau_{i}^{v}$ in the DAG-based task system, then for the $j^{\text {th }}$ jobs of the corresponding two obi-tasks, $f_{i, j}^{k} \leq a_{i, j}^{v}$.

Proof. By (7), $a_{i, j}^{k}=a_{i, j}^{1}+\Phi_{i}^{k}$, and by the definition of $R_{i}^{k}, f_{i, j}^{k} \leq$ $a_{i, j}^{k}+R_{i}^{k}$. Thus,

$$
f_{i, j}^{k} \leq a_{i, j}^{1}+\Phi_{i}^{k}+R_{i}^{k}
$$

By (7), $a_{i, j}^{v}=a_{i, j}^{1}+\Phi_{i}^{v}$, and by (10), $\Phi_{i}^{v} \geq \Phi_{i}^{k}+R_{i}^{k}$. Thus,

$$
a_{i, j}^{v} \geq a_{i, j}^{1}+\Phi_{i}^{k}+R_{i}^{k}
$$

By (11) and (12), $f_{i, j}^{k} \leq a_{i, j}^{v}$.

Property 3 shows how to compute an end-to-end response-time bound $R_{i}$.

Property 3. In the obi-task system, for each $j$, all jobs $J_{i, j}^{1}, J_{i, j}^{2}, \cdots, J_{i, j}^{n_{i}}$ finish their execution within $R_{i}$ time units after $a_{i}^{1}$, where

$$
R_{i}=\Phi_{i}^{n_{i}}+R_{i}^{n_{i}} .
$$

Proof. By (7) and the definition of $R_{i}^{v}, J_{i, j}^{v}$ finishes by time $a_{i, j}^{1}+$ $\Phi_{i}^{v}+R_{i}^{v}$. Thus, $J_{i, j}^{n_{i}}$ in particular finishes within $\Phi_{i}^{n_{i}}+R_{i}^{n_{i}}=R_{i}$ time units after $a_{i}^{1}$. Also, by (10), $\Phi_{i}^{v}+R_{i}^{v} \leq \Phi_{i}^{n_{i}}$, since $\tau_{i}^{n_{i}}$ is the single sink in $G_{i}$. Because $\Phi_{i}^{n_{i}} \leq R_{i}$, this implies that, for any $v$, 
$J_{i, j}^{v}$ finishes within $R_{i}$ time units after $a_{i}^{1}$.

Thus, a DAG-based task set can be transformed to an obi-task set with the same per-task parameters. Given these per-task parameters, a response-time bound for each obi-task can be computed by (9) for any arbitrary offset setting. Then, we can properly set the offsets for each obi-task according to (10) by considering the corresponding tasks in each DAG in topological order [11], starting with $\Phi_{i}^{1}=0$ for each source task $\tau_{i}^{1}$, by (8). By Property 2, the resulting obi-task set satisfies all requirements of the original DAGbased task system, and by Property 3 , an end-to-end response-time bound $R_{i}$ can be computed for each DAG $G_{i}$.

(Note that the response-time bound for a virtual source/sink is not computed by (9), but is zero by definition, since its WCET is zero. Any job of such a task completes in zero time as soon as it is released.)

\section{SETTING RELATIVE DEADLINES}

In the prior sections, we showed that, by applying our proposed transformation techniques, an end-to-end response-time bound for each DAG can be established, given arbitrary but fixed relativedeadline settings. That is, given $D_{i}^{v} \geq 0$ for any $i, v$, we can compute corresponding end-to-end response-time bounds (i.e., $R_{i}$ for each $i$ ) by (9), (8), (10), and (13).

Similar DAG transformation approaches have been presented previously $[13,20]$, but under the assumption that intra-task precedence constraints exist (i.e., jobs of the same task must execute in sequence). Moreover, in this prior work, per-task relative deadlines have been defined in a DAG-oblivious way. By considering the actual structure of such a DAG, it may be possible to reduce its endto-end response-time bound by setting its tasks' relative deadlines so as to favor certain critical paths.

Consider, for example, the DAG illustrated in Fig. 4. Suppose that the prior analysis yields a response-time bound of 10 for each task, as depicted within each node. The corresponding end-toend response-time bound would then be 40 and is obtained by considering the right-side path. Now, suppose that we alter the tasks' relative-deadline settings to favor the tasks along this path at the possible expense of the remaining task on the left. Further, suppose this modification changes the per-task response-time bounds to be as depicted in parentheses. Then, this modification would have the impact of reducing the end-to-end bound to 32 .

In this section, we show that the

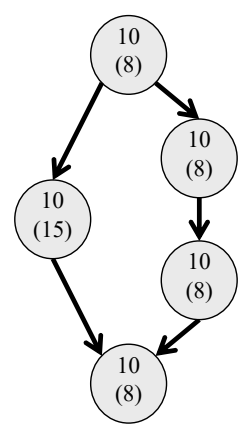

Figure 4: More highly prioritizing the right-side path in this DAG decreases its end-to-end response-time bound problem of determining the "best" relative-deadline settings can be cast as a linear-programming problem, which can be solved in polynomial time. The proposed linear program (LP) is developed in the next two subsections.

\subsection{Linear Program}

In our LP, there are three variables per task $\tau_{i}^{v}: D_{i}^{v}, \Phi_{i}^{v}$, and $R_{i}^{v}$. The parameters $T_{i}, C_{i}^{v}$, and $m_{k}$ are viewed as constants. Thus, there are $3|V|$ variables in total, where $|V|$ is the total number of tasks (i.e., nodes) across all DAGs in the system. Before stating the required constraints, we first establish the following theorem, which shows that a relative-deadline setting of $D_{x}^{y}>T_{x}$ is pointless to consider.
Theorem 2. If $D_{x}^{y}>T_{x}$, then by setting $D_{x}^{y}=T_{x}, R_{x}^{y}$, the response-time bound of task $\tau_{x}^{y}$, will decrease, and each other task's response-time bound will remain the same.

Proof. To begin, note that, by (9), $\tau_{x}^{y}$ does not impact the responsetime bounds of those tasks executing on CE pools other than $P_{x}^{y}$. Therefore, the response-time bounds $\left\{R_{i}^{v} \mid P_{i}^{v} \neq P_{x}^{y}\right\}$ for such tasks are not altered by any change to $D_{x}^{y}$.

In the remainder of the proof, we consider a task $\tau_{i}^{v}$ such that $P_{i}^{v}=P_{x}^{y}$. Let $R_{i}^{v}$ and $R_{i}^{\prime v}$ denote the response-time bounds for $\tau_{i}^{v}$ before and after, respectively, reducing $D_{x}^{y}$ to $T_{x}$. If $i=x$ and $v=y$, then by (9),

$$
\begin{aligned}
R_{x}^{y}-R_{x}^{\prime y}= & \frac{D_{x}^{y}-T_{x}}{m_{k}} \cdot \sum_{\tau_{l}^{w} \in \tau^{k}} u_{l}^{w}+ \\
& \frac{u_{x}^{y}}{m_{k}}\left(\max \left\{0, T_{x}-D_{x}^{y}\right\}-\max \left\{0, T_{x}-T_{x}\right\}\right) \\
> & \left\{\text { since } D_{x}^{y}>T_{x}\right\} \\
& 0 .
\end{aligned}
$$

Alternatively, if $i \neq x$ or $v \neq y$, then by (9),

$$
\begin{aligned}
R_{i}^{v}-R_{i}^{\prime v}= & \frac{u_{x}^{y}}{m_{k}}\left(\max \left\{0, T_{x}-D_{x}^{y}\right\}-\max \left\{0, T_{x}-T_{x}\right\}\right) \\
= & \left\{\text { since } D_{x}^{y}>T_{x}\right\} \\
& 0 .
\end{aligned}
$$

Thus, the theorem follows.

By Theorem 2, the reduction of $D_{x}^{y}$ mentioned in the theorem does not increase the response-time bound for any task. By (10), this implies that none of the offsets, $\left\{\Phi_{i}^{v}\right\}$, needs to be increased. Therefore, by Property 3, no end-to-end response-time bound increases. These properties motivate our first set of linear constraints. Constraint Set (i): For each task $\tau_{i}^{v}$,

$$
0 \leq D_{i}^{v} \leq T_{i}
$$

$2|V|$ individual linear inequalities arise from this constraint set, where $|V|$ is the total number of tasks.

Another issue we must address is that of ensuring that the offset settings, given by (10), are encoded in our LP. This gives rise to the next constraint set.

Constraint Set (ii): For each edge from $\tau_{i}^{w}$ to $\tau_{i}^{v}$ in a DAG $G_{i}$,

$$
\Phi_{i}^{v} \geq \Phi_{i}^{w}+R_{i}^{w} .
$$

There are $|E|$ distinct constraints in this set, where $|E|$ is the total number of edges in all DAGs in this system.

Finally, we have a set of constraints that are linear equality constraints.

Constraint Set (iii): With Constraints Set (i), it is clear that we can re-write (9) as follows, for each task $\tau_{i}^{v}$,

$$
\begin{aligned}
R_{i}^{v}= & \frac{1}{m_{k}}\left(D_{i}^{v} \cdot \sum_{\tau_{l}^{w} \in \Gamma_{k}} u_{l}^{w}+\sum_{\tau_{l}^{w} \in \Gamma_{k}}\left(u_{l}^{w} \cdot\left(T_{l}-D_{l}^{w}\right)\right)\right) \\
& +\max _{\tau_{l}^{w} \in \Gamma_{k}}\left\{C_{l}^{w}\right\}+\frac{m_{k}-1}{m_{k}} C_{i}^{v}
\end{aligned}
$$

where $\Gamma_{k}=\left\{\tau_{l}^{w} \mid P_{l}^{w}=\pi_{k}\right\}$. Moreover, by (8), for each DAG $G_{i}$,

$$
\Phi_{i}^{1}=0 \text {. }
$$

Constraint Set (iii) yields $|V|+|G|$ linear equations, where $|G|$ denotes the number of DAGs. 
Constraint Sets (i), (ii), and (ii) fully specify our LP, with the exception of the objective function. In this LP, there are $3|V|$ variables, $2|V|+|E|$ inequality constraints, and $|V|+|G|$ linear equality constraints.

\subsection{Objective Function}

Different objective functions can be specified for our LP that optimize end-to-end response-time bounds in different senses. Here, we consider a few examples.

Single-DAG systems. For systems where only a single DAG exists, the optimization criterion is rather clear. In order to optimize the end-to-end response-time bound of the single DAG, the objective function should minimize the end-to-end response-time bound of the only DAG, $G_{1}$. That is, the desired LP is as follows.

$$
\begin{array}{ll}
\text { minimize } & \Phi_{1}^{n_{1}}+R_{1}^{n_{1}} \\
\text { subject to } & \text { Constraint Sets (i), (ii), and (iii) }
\end{array}
$$

Multiple-DAG systems. For systems containing multiple DAGs, choices exist as to the optimization criteria to consider. We list three here.

Minimizing the average end-to-end response-time bound:

$$
\begin{array}{ll}
\operatorname{minimize} & \sum_{i}\left(\Phi_{i}^{n_{i}}+R_{i}^{n_{i}}\right) \\
\text { subject to } & \text { Constraint Sets (i), (ii), and (iii) }
\end{array}
$$

Minimizing the maximum end-to-end response-time bound:

$$
\begin{array}{ll}
\text { minimize } & Y \\
\text { subject to } & \forall i: \quad \Phi_{i}^{n_{i}}+R_{i}^{n_{i}} \leq Y \\
& \text { Constraint Sets (i), (ii), and (iii) }
\end{array}
$$

Minimizing the maximum proportional end-to-end response-time bound:

$$
\begin{array}{ll}
\operatorname{minimize} & Y \\
\text { subject to } & \forall i: \quad\left(\Phi_{i}^{n_{i}}+R_{i}^{n_{i}}\right) / T_{i} \leq Y \\
& \text { Constraint Sets (i), (ii), and (iii) }
\end{array}
$$

\section{DAG COMBINING}

In the application domain that motivates our work, the DAGs to be scheduled are typically of quite low utilizations and are defined based on a relatively small number of templates that define various computational patterns. Two DAGs defined using the same template are structurally identical: they are defined by graphs that are isomorphic, corresponding nodes from the two graphs perform identical computations, the source nodes are released at the same time, etc. Such structurally identical graphs can be combined into one graph with a reduced period and larger utilization, as long any overutilization of the underlying hardware platform is avoided. Such combining can be a very effective technique, because as the experiments presented later show, our response-time bounds tend to be proportional to periods.

We illustrate this idea with a simple example. Consider two DAGs $G_{1}$ and $G_{2}$ with a common period of $T$ that are structurally identical. A schedule of these two DAGs is illustrated abstractly at the top of Fig. 5. As illustrated at the bottom of the figure, if these two DAGs are combined, then they are replaced by a structurally identical graph, denoted here as $G_{[1,2]}$, with a period of $T / 2$. With this change, the provided response-time bounds have to be

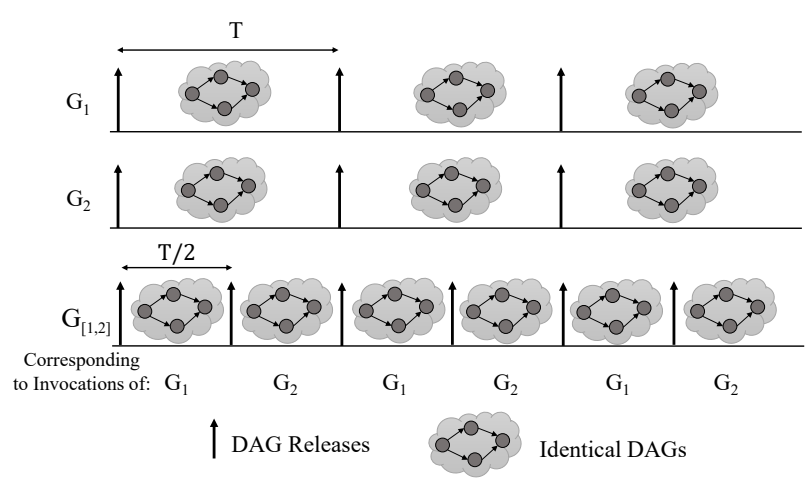

Figure 5: Illustration of DAG combining.

slightly adjusted. For example, if $G_{[1,2]}$ has a response-time bound of $R_{[1,2]}$, then this would also be a response-time bound for $G_{1}$, but that for $G_{2}$ would be $R_{[1,2]}+\frac{T}{2}$, because in combining the two graphs, the releases of $G_{2}$ are effectively shifted forward by $\frac{T}{2}$ time units. While this graph combining idea is really quite simple, the experiments presented later suggest that it can have a profound impact in the considered application domain. In particular, in that domain, per-DAG utilizations are low enough that upwards of 40 DAGs can be combined into one. Thus, the actual period reduction is not merely by a factor of $\frac{1}{2}$ but by a factor as high as $\frac{1}{40}$.

\section{EARLY RELEASING}

Transforming a DAG-based task system to a corresponding obi-task system enabled us to derive an end-to-end response-time bound for each DAG. However, such a transformation may actually cause observed end-to-end response times at runtime to increase, because the offsets introduced in the transformation may prevent a job from executing even if all of its producers have already finished. For example, in Fig. 3, $J_{1,1}^{3}$ cannot execute until time 9, even though its corresponding producer job, $J_{1,1}^{1}$, has finished by time 3 .

Observed response times can be improved under deadline-based scheduling without altering analytical response-time bounds by using a technique called early releasing [12]. When early releasing is allowed, a job is eligible for execution as soon as all of its corresponding producer jobs have finished, even if this condition is satisfied before its actual release time.

Early releasing does not affect the response-time analysis for npc-sporadic tasks presented previously [27] because this analysis is based on the total demand for processing time due to jobs with deadlines at or before a particular time instant. Early releasing does not change upper bounds on such demand, because every job's actual release time and hence deadline are unaltered by early releasing. Thus, the response-time bounds and therefore the endto-end response-time bounds previously established without early releasing still hold with early releasing.

Example 4. Considering $G_{1}$ in Fig. 1 again, Fig. 3 is a possible schedule, without early releasing, for the obi-tasks that implement $G_{1}$, as discussed earlier. When we allow early releasing, we do not change any release times or deadlines, but simply allow a job to become eligible for execution before its release time provided its producers have finished. Fig. 6 depicts a possible schedule where early releasing is allowed, assuming the same releases and deadlines as in Fig. 3. Several jobs (e.g., $J_{1,1}^{2}, J_{1,2}^{2}, J_{1,2}^{3}, J_{1,1}^{4}$, and $J_{1,2}^{4}$ ) now commence execution before their release times. As a result, observed end-to-end response times are reduced, while still retaining all response-time bounds (per-task and end-to-end). 


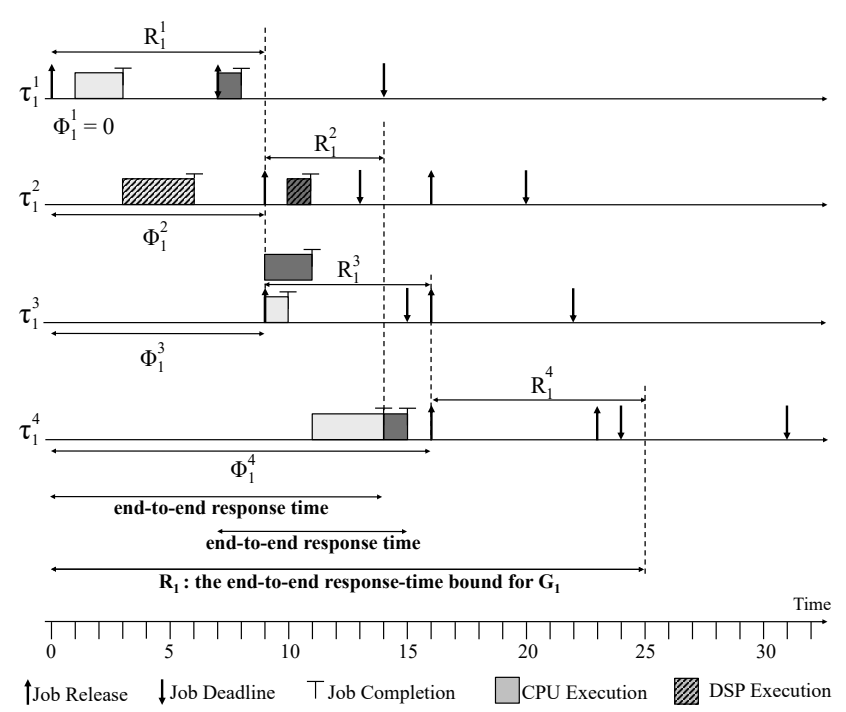

(Assume depicted jobs are scheduled alongside other jobs, which are not shown.)

Figure 6: Example schedule of the obi-tasks corresponding to the DAGbased tasks in $G_{1}$ in Fig. 1, when early releasing is allowed.

\section{CASE STUDY}

To illustrate the computational details of our analysis, we consider here a case-study system consisting of three DAGs, $G_{1}, G_{2}$, and $G_{3}$, which are specified in Fig. 7. $\pi_{1}$ is a CE pool consisting of two identical CPUs, and $\pi_{2}$ is a CE pool consisting of two identical DSPs. Thus, $m_{1}=m_{2}=2$. These three DAGs have fewer nodes and higher utilizations than typically found in our considered application domain. However, one can imagine that these graphs were obtained from combining many identical graphs of lower utilization. While it would have been desirable to consider larger graphs with more nodes, graphs from our chosen domain typically have tens of nodes, and this makes them rather unwieldy to discuss. Still, the general conclusions we draw here are applicable to larger graphs.

Utilization check. First, we must calculate the total utilization of all tasks assigned to each CE pool to make sure that neither is overutilized. We have $\sum_{\tau^{v} \in \Gamma_{1}} u_{i}^{v}=(200+100+300) / 500+$ $(133+78+197+73+5) / 1000=1.686<2$, and $\sum_{\tau_{i}^{v} \in \Gamma_{2}} u_{i}^{v}=$ $380 / 500+(16+83+242) / 1000=1.101<2$.

Virtual source/sink. Note that all DAGs have a single source and sink, except for $G_{2}$, which has two sinks. For it, we connect its two sinks to a single virtual sink $\tau_{2}^{6}$, which has a WCET of 0 and a response-time bound of 0 . We call the resulting DAG $G_{2}^{\prime}$, which is shown in Fig. 8.

Implicit deadlines. We now show how to compute response-time bounds assuming implicit deadines, i.e., $D_{1}^{v}=500$ for $1 \leq v \leq 4$, $D_{2}^{v}=1000$ for $1 \leq v \leq 5$ (the relative deadline of the virtual sink is irrelevant), and $D_{3}^{v}=1000$ for $1 \leq v \leq 3$. In order to derive an end-to-end response-time bound, we first transform the original DAG-based tasks into obi-tasks as described in Sec. 3. Next, we calculate a response-time bound $R_{i}^{v}$ for each obi-task $\tau_{i}^{v}$ by (9). The resulting task response-time bounds, $\left\{R_{i}^{v}\right\}$, are listed in Table 1 . Note that, as a virtual sink, the response-time bound for the virtual sink $\tau_{2}^{6}$ does not need to be computed by (9), but is 0 by definition. By (8) and (10), the offsets of the obi-tasks can now be computed in topological order with respect to each DAG. The resulting offsets, $\left\{\Phi_{i}^{v}\right\}$, are also shown in Table 1. Finally, by Prop-

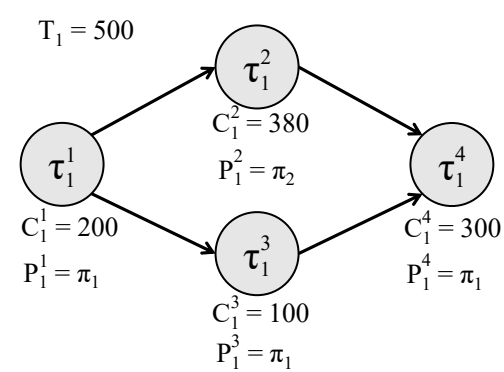

(a) $G_{1}$

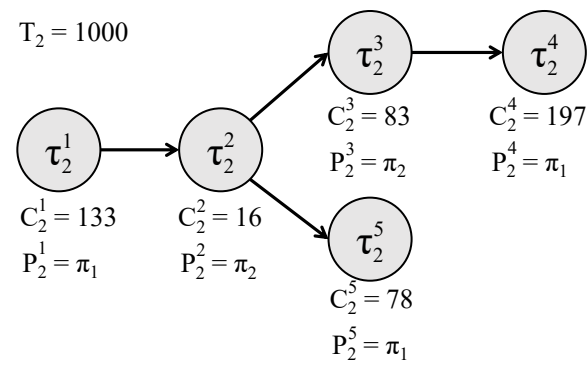

(b) $G_{2}$

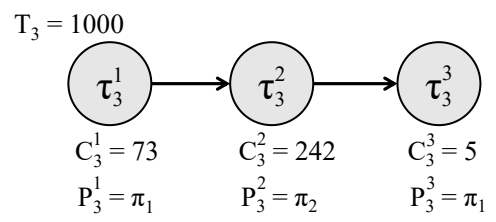

(c) $G_{3}$

Figure 7: DAGs in the case-study system. $G_{2}$ has two sinks, so to analyze it, a virtual sink $\tau_{2}^{6}$ must be added that has a WCET of 0 and a response-time bound of 0 . We show the resulting graph in Fig. 8 .

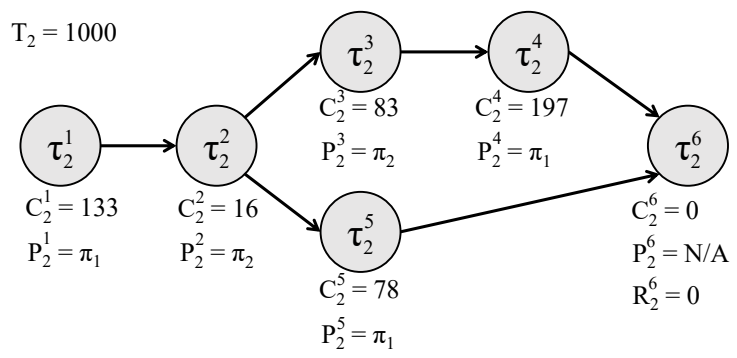

Figure 8: $G_{2}^{\prime}$, where a virtual sink is created for $G_{2}$.

erty 3, we have an end-to-end response-time bound for each DAG: $R_{1}=\Phi_{1}^{4}+R_{1}^{4}=2538.25, R_{2}=\Phi_{2}^{6}+R_{2}^{6}=4361.5$, and $R_{3}=\Phi_{3}^{3}+R_{3}^{3}=3376.5$.

LP-based deadline settings. If we use LP techniques to optimize end-to-end response-time bounds, then choices exist regarding the objective function, because our system has multiple DAGs. We consider three choices here.

Minimizing the average end-to-end response-time bound. For this choice, relative-deadline settings, obi-task response-time bounds, and obi-task offsets are as shown in Table 2(a). The resulting end-to-end response-time bounds are $R_{1}=\Phi_{1}^{4}+R_{1}^{4}=3134.5$, $R_{2}=\Phi_{2}^{6}+R_{2}^{6}=2341.2$, and $R_{3}=\Phi_{3}^{3}+R_{3}^{3}=1736.2$. 


\begin{tabular}{ccccccccccccc}
\hline$R_{1}^{1}$ & $R_{1}^{2}$ & $R_{1}^{3}$ & $\mathbf{R}_{1}^{4}$ & $R_{2}^{1}$ & $R_{2}^{2}$ & $R_{2}^{3}$ & $R_{2}^{4}$ & $R_{2}^{5}$ & $\mathbf{R}_{2}^{6}$ & $R_{3}^{1}$ & $R_{3}^{2}$ & $\mathbf{R}_{3}^{3}$ \\
\hline 821.5 & 845.25 & 771.5 & $\mathbf{8 7 1 . 5}$ & 1209.5 & 938.5 & 972 & 1241.5 & 1182 & $\mathbf{0}$ & 1179.5 & 1051.5 & $\mathbf{1 1 4 5 . 5}$ \\
\hline \hline$\Phi_{1}^{1}$ & $\Phi_{1}^{2}$ & $\Phi_{1}^{3}$ & $\mathbf{\Phi}_{1}^{4}$ & $\Phi_{2}^{1}$ & $\Phi_{2}^{2}$ & $\Phi_{2}^{3}$ & $\Phi_{2}^{4}$ & $\Phi_{2}^{5}$ & $\boldsymbol{\Phi}_{2}^{6}$ & $\Phi_{3}^{1}$ & $\Phi_{3}^{2}$ & $\boldsymbol{\Phi}_{3}^{3}$ \\
\hline 0 & 821.5 & 821.5 & $\mathbf{1 6 6 6 . 7 5}$ & 0 & 1209.5 & 2148 & 3120 & 2148 & $\mathbf{4 3 6 1 . 5}$ & 0 & 1179.5 & $\mathbf{2 2 3 1}$ \\
\hline
\end{tabular}

Table 1: Case-study task response-time bounds and obi-task offsets assuming implicit deadlines. Bold entries denote sinks.

\begin{tabular}{ccccccccccccc}
\hline \hline$D_{1}^{1}$ & $D_{1}^{2}$ & $D_{1}^{3}$ & $\mathbf{D}_{1}^{4}$ & $D_{2}^{1}$ & $D_{2}^{2}$ & $D_{2}^{3}$ & $D_{2}^{4}$ & $D_{2}^{5}$ & $\mathbf{D}_{2}^{6}$ & $D_{3}^{1}$ & $D_{3}^{2}$ & $\mathbf{D}_{3}^{3}$ \\
\hline 500 & 500 & 500 & $\mathbf{5 0 0}$ & 0 & 0 & 0 & 0 & 772.84 & $\mathbf{0}$ & 0 & 0 & $\mathbf{0}$ \\
\hline \hline$R_{1}^{1}$ & $R_{1}^{2}$ & $R_{1}^{3}$ & $\mathbf{R}_{1}^{4}$ & $R_{2}^{1}$ & $R_{2}^{2}$ & $R_{2}^{3}$ & $R_{2}^{4}$ & $R_{2}^{5}$ & $\mathbf{R}_{2}^{\mathbf{6}}$ & $R_{3}^{1}$ & $R_{3}^{2}$ & $\mathbf{R}_{3}^{3}$ \\
\hline 1034.4 & 1015.7 & 984.36 & $\mathbf{1 0 8 4 . 4}$ & 579.36 & 558.5 & 592 & 611.36 & 1203.4 & $\mathbf{0}$ & 549.36 & 671.5 & $\mathbf{5 1 5 . 3 6}$ \\
\hline \hline$\Phi_{1}^{1}$ & $\Phi_{1}^{2}$ & $\Phi_{1}^{3}$ & $\boldsymbol{\Phi}_{1}^{4}$ & $\Phi_{2}^{1}$ & $\Phi_{2}^{2}$ & $\Phi_{2}^{3}$ & $\Phi_{2}^{4}$ & $\Phi_{2}^{5}$ & $\boldsymbol{\Phi}_{2}^{6}$ & $\Phi_{3}^{1}$ & $\Phi_{3}^{2}$ & $\boldsymbol{\Phi}_{3}^{3}$ \\
\hline 0 & 1034.4 & 1049.2 & $\mathbf{2 0 5 0 . 1}$ & 0 & 579.36 & 1137.9 & 1729.9 & 1137.9 & $\mathbf{2 3 4 1 . 2}$ & 0 & 549.36 & $\mathbf{1 2 2 0 . 9}$ \\
\hline \hline
\end{tabular}

(a)

\begin{tabular}{ccccccccccccc}
\hline \hline$D_{1}^{1}$ & $D_{1}^{2}$ & $D_{1}^{3}$ & $\mathbf{D}_{1}^{4}$ & $D_{2}^{1}$ & $D_{2}^{2}$ & $D_{2}^{3}$ & $D_{2}^{4}$ & $D_{2}^{5}$ & $\mathbf{D}_{2}^{\mathbf{6}}$ & $D_{3}^{1}$ & $D_{3}^{2}$ & $\mathbf{D}_{3}^{3}$ \\
\hline 0 & 500 & 359.06 & $\mathbf{5 0 0}$ & 0 & 0 & 0 & 584.52 & 1000 & $\mathbf{0}$ & 505.63 & 1000 & $\mathbf{0}$ \\
\hline \hline$R_{1}^{1}$ & $R_{1}^{2}$ & $R_{1}^{3}$ & $\mathbf{R}_{1}^{4}$ & $R_{2}^{1}$ & $R_{2}^{2}$ & $R_{2}^{3}$ & $R_{2}^{4}$ & $R_{2}^{5}$ & $\mathbf{R}_{2}^{6}$ & $R_{3}^{1}$ & $R_{3}^{2}$ & $\mathbf{R}_{3}^{3}$ \\
\hline 642.06 & 894.75 & 894.75 & $\mathbf{1 1 1 3 . 6}$ & 608.56 & 437.5 & 471 & 1133.3 & 1424.1 & $\mathbf{0}$ & 1004.8 & 1101 & $\mathbf{5 4 4 4 . 5 6}$ \\
\hline \hline$\Phi_{1}^{1}$ & $\Phi_{1}^{2}$ & $\Phi_{1}^{3}$ & $\boldsymbol{\Phi}_{1}^{4}$ & $\Phi_{2}^{1}$ & $\Phi_{2}^{2}$ & $\Phi_{2}^{3}$ & $\Phi_{2}^{4}$ & $\Phi_{2}^{5}$ & $\boldsymbol{\Phi}_{2}^{\mathbf{6}}$ & $\Phi_{3}^{1}$ & $\Phi_{3}^{2}$ & $\boldsymbol{\Phi}_{3}^{3}$ \\
\hline 0 & 642.06 & 642.06 & $\mathbf{1 5 3 6 . 8}$ & 0 & 608.56 & 1046.1 & 1517.1 & 1128.8 & $\mathbf{2 6 5 0 . 4}$ & 0 & 1004.8 & $\mathbf{2 1 0 5 . 8}$ \\
\hline \hline
\end{tabular}

(b)

\begin{tabular}{ccccccccccccc}
\hline \hline$D_{1}^{\perp}$ & $D_{1}^{2}$ & $D_{1}^{3}$ & $\mathbf{D}_{1}^{4}$ & $D_{2}^{\perp}$ & $D_{2}^{2}$ & $D_{2}^{3}$ & $D_{2}^{4}$ & $D_{2}^{5}$ & $\mathbf{D}_{2}^{\mathbf{6}}$ & $D_{3}^{1}$ & $D_{3}^{2}$ & $\mathbf{D}_{3}^{3}$ \\
\hline 0 & 0 & 200.53 & $\mathbf{0}$ & 1000 & 0 & 253.21 & 1000 & 1000 & $\mathbf{0}$ & 1000 & 1000 & $\mathbf{1 0 0 0}$ \\
\hline \hline$R_{1}^{1}$ & $R_{1}^{2}$ & $R_{1}^{3}$ & $\mathbf{R}_{1}^{4}$ & $R_{2}^{1}$ & $R_{2}^{2}$ & $R_{2}^{3}$ & $R_{2}^{4}$ & $R_{2}^{5}$ & $\mathbf{R}_{2}^{\mathbf{6}}$ & $R_{3}^{1}$ & $R_{3}^{2}$ & $\mathbf{R}_{3}^{3}$ \\
\hline 679.95 & 798.99 & 798.99 & $\mathbf{7 2 9 . 9 5}$ & 1489.4 & 616.99 & 789.89 & 1521.4 & 1461.9 & $\mathbf{0}$ & 1459.4 & 1280.5 & $\mathbf{1 4 2 5 . 4}$ \\
\hline \hline$\Phi_{1}^{1}$ & $\Phi_{1}^{2}$ & $\Phi_{1}^{3}$ & $\mathbf{\Phi}_{1}^{4}$ & $\Phi_{2}^{1}$ & $\Phi_{2}^{2}$ & $\Phi_{2}^{3}$ & $\Phi_{2}^{4}$ & $\Phi_{2}^{5}$ & $\mathbf{\Phi}_{2}^{6}$ & $\Phi_{3}^{1}$ & $\Phi_{3}^{2}$ & $\boldsymbol{\Phi}_{3}^{3}$ \\
\hline 0 & 679.95 & 679.95 & $\mathbf{1 4 7 8 . 9}$ & 0 & 1489.4 & 2106.4 & 2896.3 & 2552.7 & $\mathbf{4 4 1 7 . 8}$ & 0 & 1508.3 & $\mathbf{2 8 3 5 . 6}$ \\
\hline \hline
\end{tabular}

(c)

Table 2: Case-study relative-deadline settings, obi-task response-time bounds, and obi-task offsets when using linear programming to (a) minimize average end-to-end response-time bounds, (b) minimize maximum end-to-end response-time bounds, and (c) minimize maximum proportional end-to-end responsetime bounds. Bold entries denote sinks.

Minimizing the maximum end-to-end response-time bound. For this choice, relative-deadline settings, obi-task response-time bounds, and obi-task offsets are as shown in Table 2(b). The resulting end-to-end response-time bounds are $R_{1}=\Phi_{1}^{4}+R_{1}^{4}=2650.4$, $R_{2}=\Phi_{2}^{6}+R_{2}^{6}=2650.4$, and $R_{3}=\Phi_{3}^{3}+R_{3}^{3}=2650.4$.

Minimizing the maximum proportional end-to-end responsetime bound. For this choice, relative-deadline settings, obi-task response-time bounds, and obi-task offsets are as shown in Table 2(c). The resulting end-to-end response-time bounds are $R_{1}=$ $\Phi_{1}^{4}+R_{1}^{4}=2208.9, R_{2}=\Phi_{2}^{6}+R_{2}^{6}=4417.8$, and $R_{3}=$ $\Phi_{3}^{3}+R_{3}^{3}=4261.0$.

Early releasing. As discussed in Sec. 7, early releasing can improve observed response times without compromising responsetime bounds. The value of allowing early releasing can be seen in the results reported in Table 3. This table gives the largest observed end-to-end response time of each DAG in Fig. 7, assuming implicit deadlines with and without early releasing, in a schedule that was simulated for 50,000 time units. Analytical bounds are shown as well.

\begin{tabular}{c|ccc}
\hline & $G_{1}$ & $G_{2}$ & $G_{3}$ \\
\hline Early releasing & 1006 & 897 & 453 \\
\hline No early releasing & 1966.75 & 3536.25 & 2586.0 \\
\hline Bounds & 2538.25 & 4361.5 & 3376.5
\end{tabular}

Table 3: Observed end-to-end response times with/without early releasing and analytical end-to-end response-time bounds for the implicit-deadline setting.

\section{SCHEDULABILITY STUDIES}

In this section, we expand upon the specific case study just described by considering general schedulability trends seen in experiments involving randomly generated task systems.

\subsection{Improvements Enabled by Basic Tech- niques}

We first consider the improvements enabled by the basic techniques covered in Secs. 4 and 5 that underlie our work: allowing intra-task parallelism as provided by the npc-sporadic task model, and determining relative-deadline settings by solving an LP.

Random system generation. In our experiments, we considered a heterogeneous platform comprised of three CE pools, each consisting of eight identical CEs. Each pool was assumed to have the same total utilization. We considered all choices of total per-pool utilizations in the range $[1,8]$ in increments of 0.5 .

We generated DAG-based task systems using a method similar to that used by others $[4,17]$. These systems were generated by first specifying the number of DAGs in the system, $N$, and the number of tasks per DAG, $n$. For each considered pair $N$ and $n$, we randomly generated 50 task-system structures, each comprised of $N$ DAGs with $n$ nodes. Each node in such a structure was randomly assigned to one of the CE pools, and for each DAG in the structure, one node was designated as its source, and one as its sink. Further, each pair of internal nodes (not a source or a sink) was connected 


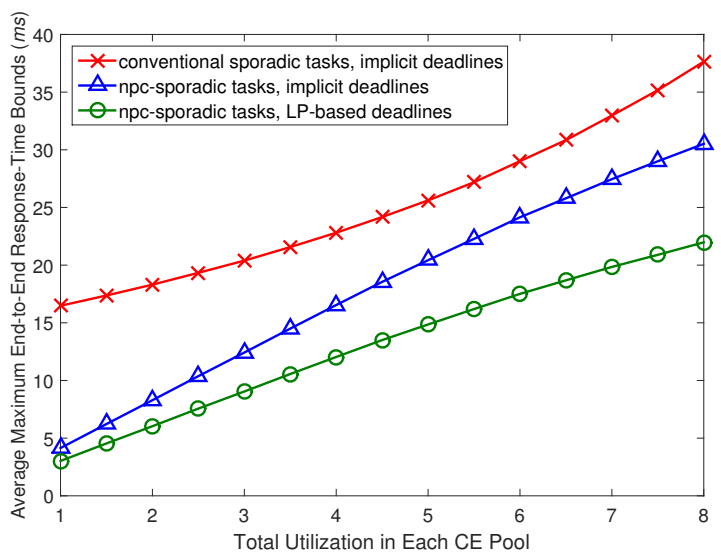

Figure 9: AMERBs as a function of total utilization in each CE pool in the case where each task set has five DAGs, 20 tasks per DAG, and edgeP rob $=0.5$.

by an edge with probability edgeProb, a settable parameter. Such an edge was directed from the lower-indexed node to the higherindexed node, to preclude cycles. Finally, an edge was added from the source to each internal node with no incoming edges, and to the sink from each internal node with no outgoing edges.

For each considered per-pool untilization and each generated task-system structure, we randomly generated 50 actual task systems by generating task utilizations using the MATLAB function randfixedsum() [25]. According to the application domain that motivates this work, ${ }^{2}$ we defined each DAG's period to be $1 \mathrm{~ms}$. (A task's WCET is determined by its utilization and period.) For each considered value of $N, n$, and total per-pool utilization (one point in one of our graphs), we considered 50 (task system structures) $\times 50$ (utilizations $)=2,500$ task sets.

Comparison setup. We compared three strategies: (i) transforming to a conventional sporadic task system and using implicit relative deadlines, which is a strategy used in prior work on identical platforms [20]; (ii) transforming to an npc-sporadic task system and using implicit relative deadlines; and (iii) transforming to an npc-sporadic task system and using LP-based relative deadlines. When applying our LP techniques, we chose the objective function that minimizes the maximum end-to-end response-time bound. Although an identical platform was assumed in [20], the techniques from that paper can be extended to heterogeneous platforms in a similar way to this paper.

Results. In all cases that we considered, the two evaluated techniques improved end-to-end response-time bounds, often significantly. Due to space constraints, we present here only the case where $N=5, n=20$, and edgeProb $=0.5$. For each generated task set, we recorded the maximum end-to-end responsetime bound among its five DAGs. For each given total per-pool utilization point, we report here the average of the maximum endto-end response-time bounds among the 2,500 task sets generated for that point. We call this metric the average maximum end-to-end response-time bound (AMERB). Fig. 9 plots AMERBs as a function of total per-CE-pool utilization. As seen, the application of both techniques reduced AMERBs by $39.42 \%$ to $81.65 \%$.

In addition to this plot, we also considered other cases with different values for $N, n$, and edgeProb. These other results show

\footnotetext{
${ }^{2}$ In applications usually considered in the real-time-systems community, much larger periods are the norm. The considered domain is quite different.
}

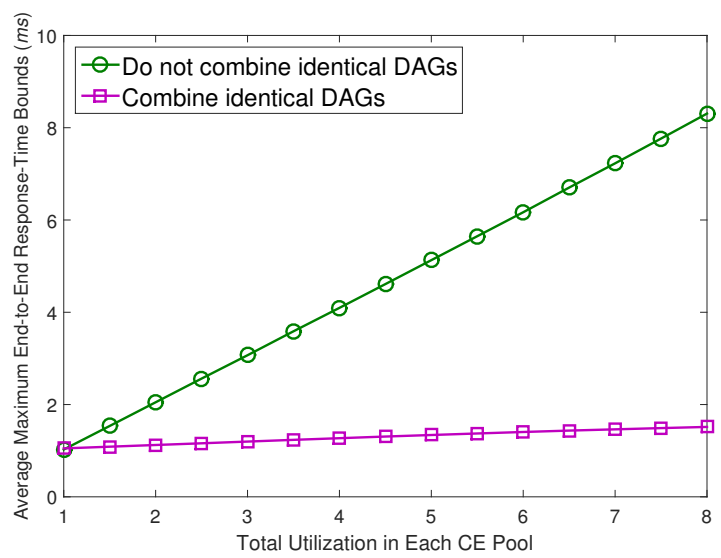

Figure 10: AMERBs as a function of total utilization in each CE pool in the case where the number of identical DAGs per template is fixed to 40 .

similar trends and can be found in an online appendix (available at http://cs.unc.edu/ anderson/papers.html).

\subsection{Improvements Enabled by DAG Combin- ing}

As mentioned in Sec. 6, in the application domain that motivates our work, DAGs are usually defined using several well-defined computational templates, and as a result, many identical DAGs will exist. We proposed the technique of DAG combining in Sec. 6 to exploit this fact to further reduce response-time bounds. We now discuss schedulability experiments that we conducted to evaluate this technique.

Random system generation. We employed a process of randomly generating systems that is similar to that discussed in Sec. 9.1, except that, instead of generating task-system structures comprised of $N$ DAGs, we generated structures comprised of $N$ templates. Additionally, we introduced a new parameter $K$ that indicates the number of identical DAGs per template. A period of $1 \mathrm{~ms}$ was still associated with each DAG.

Comparison setup. We compared two strategies: (i) do no combining, and compute end-to-end response-time bounds assuming $N \cdot K$ independent DAGs; (ii) combine identical DAGs, and compute end-to-end response-time bounds assuming $N$ DAGs, making adjustments as discussed in Sec. 6 to obtain actual response-time bounds for the DAGs that were combined. Under both strategies, the general techniques evaluated in Sec. 9.1 were applied.

Results. In all cases that we considered, the DAG combining technique improved end-to-end response-time bounds significantly. Due to space constraints, we present here only the case where each system has five templates, each of which has 20 nodes, and edgeProb $=0.5$. Other results can be found online. For the considered case, Fig. 10 plots AMERBs as a function of total perpool utilization, when the number of identical DAGs per template is fixed to 40 (this number is close to what would be expected in the application domain that motivates this work). Note that the AMERBs in Fig. 10 are much lower than those in Fig. 9, even before applying the DAG combining technique. This is because the systems considered in Fig. 10 have far more DAGs than those in Fig. 9. As a result, for each given total per-pool utilization, the systems in Fig. 10 have much lower per-DAG and per-task utilizations. As also seen in Fig. 10, when DAG combining is applied, the AMERBs are not very much influenced by the total per-CEpool utilization. That is because DAG combining resulted in quite 


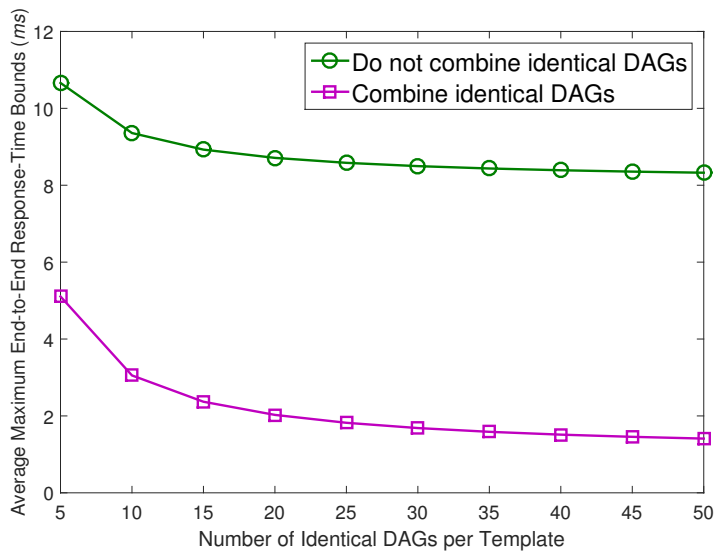

Figure 11: AMERBs as a function of the number of identical DAGs per template in the case where total utilization in each CE pool is fixed to eight.

small response-time bounds by (9), so total end-to-end bounds were mainly impacted by the introduced shifting, rather than total perCE-pool utilization. Also, Fig. 11 plots AMERBs as a function of the number of identical DAGs per template, when every CE pool is fully utilized (i.e., the total utilization of each pool is eight). In this case, the AMERB metric was calculated over all task sets that have the same number of identical DAGs per template.

According to our industry partners, in the considered application domain, a DAG's end-to-end response-time bound should typically be at most $2.35 \mathrm{~ms}$. As observed in Fig. 10, in the absence of DAG combining, AMERBs in this experiment were as high as $8.2 \mathrm{~ms}$. However, the introduction of DAG combining enabled a drop to less than $2.0 \mathrm{~ms}$, even when the platform was fully utilized. This demonstrates that DAG combining - as simple as it may seem-can have a powerful impact in the targeted domain.

\section{CONCLUSION}

We presented task-transformation techniques to provide endto-end response-time bounds for DAG-based tasks implemented on heterogenous multiprocessor platforms where intra-task parallelism is allowed. We also presented an LP-based method for setting relative deadlines and a DAG combining technique that can be applied to improve these bounds. We evaluated the efficacy of these results by considering a case-study task system and by conducting schedulability studies. To our knowledge, this paper is the first to present end-to-end response-time analysis for the considered context. In future work, we intend to extend these techniques to deal with synchronization requirements and to incorporate methods for limiting interference caused by contention for shared hardware components such as caches, memory banks, etc.

Acknowledgments: We thank Alan Gatherer, Lee McFearin, and Peter Yan for bringing the problem studied in this paper to our attention and for answering many questions regarding cellular base stations.

\section{References}

[1] H. Ali, B. Akesson, and L. Pinho. Generalized extraction of real-time parameters for homogeneous synchronous dataflow graphs. In 23rd PDP, 2015.

[2] P. Axer, S. Quinton, M. Neukirchner, R. Ernst, B. Dobel, and H. Hartig. Response-time analysis of parallel fork-join workloads with real-time constraints. In 25th ECRTS, 2013.

[3] R. Bajaj and D. Agrawal. Scheduling multiple task graphs in heterogeneous distributed real-time systems by exploiting schedule holes with bin packing techniques. IEEE Transactions on Parallel and Distributed Systems, 15(2):107-118, 2004.

[4] S. Baruah. Improved multiprocessor global schedulability analysis of sporadic DAG task systems. In 26th ECRTS, 2014.

[5] S. Baruah. The federated scheduling of constrained-deadline sporadic DAG task systems. In 18th DATE, 2015.

[6] S. Baruah. Federated scheduling of sporadic DAG task systems. In 29th IPDPS, 2015.

[7] S. Baruah, V. Bonifaci, A. Marchetti-Spaccamela, L. Stougie, and A. Wiese. A generalized parallel task model for recurrent real-time processes. In 33rd RTSS, 2012.

[8] big.LITTLE Processing. http://www.arm.com/products/processors/technologies/biglittleprocessing.php.

[9] V. Bonifaci, A. Marchetti-Spaccamela, S. Stiller, and A. Wiese. Feasibility analysis in the sporadic DAG task model. In 25th ECRTS, 2013.

[10] H. Chwa, J. Lee, K. Phan, A. Easwaran, and I. Shin. Global EDF schedulability analysis for synchronous parallel tasks on multicore platforms. In 25th ECRTS, 2013.

[11] T. Cormen, C. Leiserson, R. Rivest, and C. Stein. Introduction to Algorithms. The MIT Press, 2009.

[12] U. Devi. Soft Real-Time Scheduling on Multiprocessors. $\mathrm{PhD}$ thesis, University of North Carolina, Chapel Hill, NC, 2006.

[13] G. Elliott, N. Kim, J. Erickson, C. Liu, and J. Anderson. Minimizing response times of automotive dataflows on multicore. In 20th RTCSA, 2014.

[14] J. Erickson and J. Anderson. Response time bounds for G-EDF without intra-task precedence constraints. In 15th OPODIS, 2011.

[15] J. Fonseca, V. Nelis, G. Raravi, and L. Pinho. A multi-DAG model for real-time parallel applications with conditional execution. In 30th SAC, 2015.

[16] T. Grandpierre, C. Lavarenne, and Y. Sorel. Optimized rapid prototyping for real-time embedded heterogeneous multiprocessors. In 7th CODES, 1999.

[17] J. Li, K. Agrawal, C. Lu, and C. Gill. Analysis of global EDF for parallel tasks. In 25th ECRTS, 2013.

[18] J. Li, A. Saifullah, K. Agrawal, C. Gill, and C. Lu. Analysis of federated and global scheduling for parallel real-time tasks. In 26th ECRTS, 2014.

[19] J. Li, A. Saifullah, K. Agrawal, C. Gill, and C. Lu. Capacity augmentation bound of federated scheduling for parallel DAG tasks. Technical report, Washington University in St Louis, 2014.

[20] C. Liu and J. Anderson. Supporting soft real-time DAG-based systems on multiprocessors with no utilization loss. In 31st RTSS, 2010.

[21] C. Maia, M. Bertogna, L. Nogueira, and L. Pinho. Global EDF schedulability analysis for synchronous parallel tasks on multicore platforms. In 22nd RTNS, 2014.

[22] A. Melani, M. Bertogna, V. Bonifaci, A. MarchettiSpaccamela, and G. Buttazzo. Response-time analysis of conditional DAG tasks in multiprocessor systems. In 27th ECRTS, 2015.

[23] A. Parri, A. Biondi, and M. Marinoni. Response time analysis for G-EDF and G-DM scheduling of sporadic DAG-tasks with arbitrary deadline. In 23rd RTNS, 2015.

[24] A Saifullah, K. Agrawal, C. Lu, and C. Gill. Multi-core realtime scheduling for generalized parallel task models. In $32 \mathrm{nd}$ RTSS, 2011.

[25] R. Stafford. Random vectors with fixed sum. http://www. mathworks.com/matlabcentral/fileexchange/9700-randomvectors-with-fixed-sum.

[26] G. Stavrinides and H. Karatza. Scheduling multiple task graphs in heterogeneous distributed real-time systems by exploiting schedule holes with bin packing techniques. Simulation Modelling Practice and Theory, 19(1):540-552, 2011.

[27] K. Yang and J. Anderson. Optimal GEDF-based schedulers that allow intra-task parallelism on heterogeneous multiprocessors. In ESTIMedia, 2014. 